\title{
An eight-year retrospective analysis of antenatal screening results for syphilis: is it still cost effective?
}

\author{
Tugba Ensari, Ayse Kirbas, Ayse Seval Ozgu-Erdinc, Sibel Gokay Saygan, Salim Erkaya, Dilek Uygur, \\ Nuri Danisman
}

Zekai Tahir Burak Women Health Care, Training and Research Hospital, Ankara, Turkey

\begin{abstract}
Introduction: This study aimed to document the prevalence of syphilis among pregnant women in Turkey.

Methodology: In this retrospective cohort study, a total of 63,276 sera obtained between January 2007 and June 2014 from women who were routinely screened for syphilis as a part of antenatal care at a tertiary referral hospital in Turkey were analyzed. Serological screening was done with the rapid plasma reagin (RPR) test on venous blood samples. Treponema pallidum hemagglutination assay (TPHA) was the confirmation test for the diagnosis of syphilis in patients who had positive results in the screening test.

Results: Between 2007 and the first six months of 2014, 41 RPR+ and only five confirmed syphilis-positive patients were determined. The syphilis seroprevalence rate was $0.0648 \%$. Within these years, there was no case of congenital syphilis detected in the hospital.

Conclusion: As there is evidence of effective screening of syphilis contributing to the effective treatment and prevention of adverse outcomes, routine antenatal screening of syphilis is recommended. The rationale depends on the consideration of the serious results of not treating the disease and the cost effectiveness of screening.
\end{abstract}

Key words: syphilis; prevalence; pregnancy; antenatal care.

J Infect Dev Ctries 2015; 9(9):1011-1015. doi:10.3855/jidc.6064

(Received 10 October 2014 - Accepted 20 March 2015)

Copyright (C) 2015 Ensari et al. This is an open-access article distributed under the Creative Commons Attribution License, which permits unrestricted use, distribution, and reproduction in any medium, provided the original work is properly cited.

\section{Introduction}

Syphilis has remained one of the major public health problems, especially in developing countries. The World Health Organization (WHO) reported that more than two million pregnant women, mostly from low-income and middle-income countries, have active syphilis every year [1].

Treponema pallidum is able to cross through the placenta and infect the fetus from the sixth gestational week, and the transmission risk increases with time. Clinical manifestations are not apparent until 16 weeks of gestation, when fetal immunocompetence develops. Transmission may also occur during delivery [2-4].

Antenatal syphilis screening is highly recommended by reproductive healthcare programs [5]. The goal of antenatal screening for syphilis is primarily to detect pregnant women with congenitally transferable syphilis. Congenital syphilis is a multiorgan infection resulting in stillbirth (24.7\%), premature birth $(24.7 \%)$, neonatal death $(12.3 \%)$, severe illness in the infancy period (4.1\%), and infant death (11.2\%) [6-9]. Congenital syphilis can be prevented with early detection and adequate treatment [10].

Traditionally, congenital syphilis prevention relied on pregnancy screening of primary and secondary syphilis [11,12]. Importantly, the majority of women with syphilis are asymptomatic, and therefore serological screening is the only method of diagnosis. Treponema pallidum cannot be cultured. Also, identification of the spirochete is only possible in patients with lesions of primary or secondary syphilis [13]. Therefore, serologic testing is the preferred method to diagnose syphilis for screening purposes or in patients with suspected disease [8].

The Centers for Disease Control and Prevention (CDC) recommends universal serological syphilis screening during early pregnancy, which should be repeated in the third trimester and at delivery for populations where syphilis prevalence is high or patients are at high risk [14]. All women who experience stillbirth after 20 weeks of gestation should be tested for syphilis [14].

The present study aimed to determine the syphilis prevalence among patients attending the outpatient 
clinic of a tertiary referral center in Turkey and, in light of the literature, to present a basic discussion of the rationale for routine antenatal syphilis screening.

\section{Methodology}

This was a retrospective cohort study based on patient records obtained from the outpatient clinic of the Zekai Tahir Burak Women Health Care, Training and Research Hospital in Ankara, Turkey.

The institutional review board and the research ethics committee approved the study protocol.

Serological syphilis screening was performed free of charge on all pregnant women as a part of routine antenatal care at their first hospital visit during pregnancy. During this period, 63,276 sera were obtained between 2007 and the first six months of 2014 for syphilis testing at the outpatient clinic of a tertiary referral center. Serological screening was performed using the rapid plasma reagin (RPR) test. Treponema pallidum hemagglutination assay (TPHA) was used to confirm positive screening results for syphilis for the diagnosis of patients with positive results [15]. Two technicians reviewed each result independently during the whole process.

The data were presented as number with percentage for categorical variables. SPSS (Statistical Package for the Social Sciences) version 21.0 was used for the statistical calculations. The data were summarized as mean \pm standard deviation and median (minimum-maximum).

\section{Results}

Among 63,276 patients, the mean age was 26.5 years (range, 11-49 years). The incidence of positive syphilis serology was found to be $0.0648 \%$ (41 patients), and five confirmatory tests were positive $(0.0079 \%)$. During the study period, there were
150,661 deliveries of 154,176 babies in the hospital. All five of the TPHA-positive pregnant women diagnosed with syphilis were treated with benzathine penicillin and all except one gave birth to normal babies without any signs of congenital syphilis. One pregnancy with a TPHA-positive pregnant patient is still in antenatal follow-up and is being treated (Table 1).

\section{Discussion}

Prevention of congenital syphilis with antenatal screening and treatment has been well established $[16,17]$. Our hospital is a tertiary healthcare referral institution that serves a population with a varied ethnic and socioeconomic base and is one of the biggest women's health care centers in Turkey. We found that the prevalence of syphilis seropositivity was very low in our population. We determined only five confirmed syphilis cases among 63,276 pregnant women between January 2007 and June 2014 (0.0079\%). The present report presents the data of the largest pregnant population from Turkey to date. Although in Turkey there is no health policy for screening for syphilis and there are different approaches among hospitals, in our hospital we routinely screen all patients at the first antenatal visit.

During the study interval, there was no newborn diagnosed with congenital syphilis. At the beginning of the study, we did not expect such a low prevalence of syphilis. It may be due to regional or socioeconomic characteristics of the population screened, but it is still important in order to discuss the cost effectiveness of antenatal syphilis screening because of the size of the study population.

There is a lack of population-based data regarding the exact prevalence of syphilis in Turkey. Aktürk et al. in their 2009 report referring to Ministry of Health

Table 1. Seropositivity rates of syphilis over years

\begin{tabular}{cccccc}
\hline & $\mathbf{N}$ & RPR+ & \% & TPHA+ & \% \\
\hline 2007 & 4,530 & 13 & 0.2870 & 1 & 2 \\
2008 & 5,990 & 13 & 0.2170 & 0 & 0.0221 \\
2009 & 7,018 & 8 & 0.1140 & 0 & 0.0000 \\
2010 & 8,934 & 1 & 0.0112 & 0 & 0.0000 \\
2011 & 10,645 & 2 & 0.0188 & 0.0000 & 0.0000 \\
2012 & 10,949 & 0 & 0.0000 & 0.0092 \\
2013 & 10,905 & 1 & 0.0697 & 0.0232 \\
2014 & 4,305 & 3 & $\mathbf{0 . 0 6 4 8}$ & $\mathbf{0 . 0 0 7 9}$ \\
Total & $\mathbf{6 3 , 2 7 6}$ & $\mathbf{4 1}$ & & $\mathbf{5}$ \\
\hline
\end{tabular}


records, gave a prevalence of $0.00495 \%$ in the whole country, including men [18]. Dilek et al. evaluated 39,002 blood donors over a nine-year period for various infectious diseases, such hepatitis $B$, hepatitis C, syphilis, and HIV seropositivity. Of the 39,002 donors, 16,601 (42\%) were females. Although the overall positivity rate of the Venereal Disease Research Laboratory (VDRL) test was $0.057 \%$, it was not calculated for female donors separately [19]. Oner et al. published a descriptive study conducted among 30,716 blood donors in the Mediterranean region of Turkey. There were no positive VDRL results detected among 1,129 female donors in the study [20]. There are two studies from Turkey that provide syphilis seropositivity rates of pregnant women; Demirel et al. reported a seroprevalence of syphilis of $0.1 \%$ among 916 patients from the eastern region of Turkey [21], and Nas et al. found only one VDRL+ patient among 3,050 pregnant women in 1999 but could not confirm the diagnosis of syphilis [22]. According to these few national publications focusing on prevalence determination, although Turkey takes its place among the countries with a very low prevalence of syphilis, this may not remain so indefinitely. This issue may be a reason for discussing the cost effectiveness of syphilis screening in Turkey.

Congenital syphilis is now rare in wealthy countries; however, adverse pregnancy outcome results are still important public health problems in many underdeveloped or developing areas. In 1993, the World Development Report first determined that syphilis screening during the antenatal period is one of the most cost-effective actions that can be taken to improve children's health [23]. The WHO states that the biggest problem is inadequate screening of syphilis in regions where the infection has a higher prevalence [23]. A WHO initiative to eliminate mother-to-child transmission of syphilis aims for a $\geq 90 \%$ pregnant women screening rate and to provide adequate treatment for $\geq 90 \%$ of seropositive women by 2015 [15]. Estimates from the WHO in 2008 revealed that nearly 1.4 million pregnant women had a probable active syphilis infection and were at risk of transmitting the disease perinatally to their unborn children [24]. Despite highly recommended preventive health care programs, one-third of women are not tested for syphilis during antenatal care management, and a large number in the population will remain untreated $[24,25]$. In the WHO/UNAIDS/UNICEF 2011 progress report on the global HIV/AIDs response, low- and middle-income countries reported syphilis testing as part of antenatal care (ANC) in $68 \%$ of women; however, this number may not reflect the most objective findings because of the lack of an organized reporting system [26]. In the present report, there was no available data from Turkey concerning the proportion of women attending ANC who were screened for syphilis at the initial visit. We believe that the data of 63,276 pregnant patients reflects a large population and provides important information.

Access to antenatal care is one of the agreed milestones of progress towards the Millennium Development Goals (MDG). Infections, as well as prematurity and birth asphyxia, contribute to $80 \%$ of neonatal deaths, and it has been shown that improving healthcare programs (including syphilis screening) beginning from the antenatal period would avert neonatal and infant deaths in order to attain the fourth MDG [27,28]. For the worldwide elimination of mother-to-child transmission of syphilis, one of the important steps defined in the report was "early antenatal care", which was found in a meta-analyses to decrease adverse pregnancy outcomes caused by syphilis infection of the mother when compared to pregnant women seen at the third trimester (odds ratio, 2.24) [29]. In some countries where seropositivity prevalence rates of syphilis are high or new seroconversion cases are detected during ongoing pregnancies, and where the population is composed of various ethnic and social characteristics, revision of the screening protocol is a question for antenatal care management policies. Some countries, especially in Europe, which are targets of migration flow, are also subject to a rise in the prevalence of syphilis. In these countries, applying a second screening test in the last trimester of pregnancy has been proposed [30].

Studies aiming to determine the most optimal test for syphilis are still in progress. There are two commonly used serological tests for syphilis: the nontreponemal and the treponemal tests. Serologic screening with a non-treponemal test is used to identify patients with possible untreated syphilis infection. To date, non-treponemal tests are in use that have acceptable accuracy, sensitivity, and cost [31]. Non-treponemal tests are the VDRL and RPR tests. These tests are relatively inexpensive and sensitive (86\%-100\% for RPR and $78 \%-100 \%$ for VDRL), making them useful as screening tests, but they require at least a basic laboratory. A positive screening test is confirmed by one of the treponemal tests: TPHA, fluorescent-treponemal antibody-absorbed test (FTA$\mathrm{ABS}$ ), and microhemagglutination test for antibodies to T. pallidum (MHA-TP). TPHA and enzyme immunoassays (EIAs) are more expensive and require 
a reference laboratory [14]. The rate of false-positive results is lower in treponemal tests than in nontreponemal tests. Previous studies have demonstrated that several conditions, such as tumors, infectious diseases, and autoimmune diseases can cause biological false-positive (BFP) reactivity. Also, elderly people and pregnant women are at risk of falsepositive results because of higher autoantibody titers [32,33].

Many studies calculating the cost effectiveness of screening compared to costs of treating infants with congenital syphilis, severe adverse outcomes, and disability-adjusted life years (DALY), found that antenatal screening of syphilis is highly cost effective, even in areas with very low prevalence rates [34-36]. When positive-screened pregnant women are treated adequately, the effectiveness of treatment is assumed to be $100 \%$ for prevention of adverse outcomes. This high rate of cure after treatment makes the decision to take action in public health policies very easy.

\section{Conclusions}

Since there is evidence of effective screening of syphilis contributing to the effective treatment and prevention of adverse outcomes during pregnancy, such as severe illness in the infancy period and perinatal death, and given the acceptable costs of screening tests, routine antenatal screening for syphilis is suggested. The rationale depends on the comparison of the serious results of not treating and the cost effectiveness of screening.

\section{References}

1. Schmid GP, Stoner BP, Hawkes S, Broutet N (2007) The need and plan for global elimination of congenital syphilis. Sex Transm Dis 34: S5-S10.

2. Gust DA, Levine WC, St Louis ME, Braxton J, Berman SM (2002) Mortality associated with congenital syphilis in the United States, 1992-1998. Pediatrics 109: E79-E90.

3. Goldenberg RL, Thompson C (2003) The infectious origins of stillbirth. Am J Obstet Gynecol 189: 861-873.

4. Bittencourt AL, Garcia AG (2002) Pathogenesis and pathology of hematogenous infections of the fetus and newborn. Pediatr Pathol Mol Med 21: 353-399.

5. Hawkes S, Matin N, Broutet N, Low N (2011) Effectiveness of interventions to improve screening for syphilis in pregnancy: a systematic review and meta-analysis. Lancet Infect Dis 11: 684-691.

6. Watson-Jones D, Changalucha J, Gumodoka B, Weiss H, Rusizoka M, Ndeki L, Whitehouse A, Balira R, Todd J, Ngeleja D Ross D, Buvé A, Hayes R, Mabey D (2002) Syphilis in pregnancy in Tanzania. I. Impact of maternal syphilis on outcome of pregnancy. J Infect Dis 186: 940-947.
7. McDermott J, Steketee R, Larsen S, Wirima J (1993) Syphilis-associated perinatal and infant mortality in rural Malawi. Bull World Health Organ 71: 773-780.

8. Romanowski B, Sutherland R, Fick GH, Mooney D, Love EJ (1991) Serologic response to treatment of infectious syphilis. Ann Intern Med 114: 1005-1009.

9. Louis M (1996) Strategies for syphilis prevention in the 1990s. Sex Transm Dis 23: 58-67.

10. Peeling RW, Ye H (2004) Diagnostic tools for preventing and managing maternal and Congenital syphilis:an overview. Bull World Health Organ 82: 439-446.

11. Blank S, McDonnell DD, Rubin SR, Neal JJ, Brome MW, Masterson MB, Greenspan JR (1997) New approaches to syphilis control. Finding opportunities for syphilis treatment and congenital syphilis prevention in a women's correctional setting. Sex Transm Dis 24: 218-226.

12. Lago EG, Rodrigues LC, Fiori RM, Stein AT (2004) Congenital syphilis: identification of two distinct profiles of maternal characteristics associated with risk. Sex Transm Dis 31: 33-37.

13. World Health Organization (1999) Laboratory tests for detection of reproductive tract infections. Available: http://www.wpro.who.int/publications/pub_9290611480/en. Accessed 10 October 2014.

14. Centers for Disease Control and Prevention (2006) Sexually transmitted diseases treatment guidelines, 2006. MMWR 55: 1-94.

15. World Health Organization (2007) The global elimination of congenital syphilis: rationale and strategy for action. Available:

http://apps.who.int/iris/bitstream/10665/43782/1/9789241595 858_eng.pdf. Accessed: July 24, 2013.

16. Connor N, Roberts J, Nicoll A (2000) Strategic options for antenatal screening for syphilis in the United Kingdom: a cost effectiveness analysis. J Med Screen 7: 7-13.

17. Hawkes S, Miller S, Reichenbach L, Nayyar A, Buses K (2004) Antenatal syphilis control: people, programmes, policies and politics. Bull World Health Organ 82: 417-423.

18. Akturk AS, Bilen N, Demirsoy EO, Kiran R (2009) The increase rates of syphilis in Turkey in the beginning of the third millennium. J Eur Acad Dermatol Venereol 23: 12091210.

19. Dilek İ, Demir C, Bay A, Akdeniz H, Oner A (2007) Seropositivity rates of $\mathrm{HBsAg}$, anti-HCV, anti-HIV and VDRL in blood donors in Eastern Turkey. Turk J Hematol 24: 4-7.

20. Oner S YG, Şaşmaz CT, Kurt AÖ, Buğdaycı R (2011) Hepatitis B, hepatitis C, HIV, and VDRL seroprevalence of blood donors in Mersin, Turkey. Turk J Med Sci 41: 335-341.

21. Demirel Y, Duran B, Toktamis A, Erden O, Cetin M (2004) Seroprevalence of syphilis, hepatitis B and C, and human immunodeficiency virus infections among women. Saudi Med J 25: 2037-2038.

22. Nas T, Taner MZ, Yildiz A (1999) Seroprevalence of syphilis, human immunodeficiency virus type-1, and hepatitis $\mathrm{B}$ virus infections among pregnant women in Turkey. Int $\mathrm{J}$ Gynaecol Obstet 66: 171-172.

23. Rivera R, Chi IC, Farr G (1993) The intrauterine device in the present and future. Curr Opin Obstet Gynecol 5: 829-832.

24. Newman L, Kamb M, Hawkes S, Gomez G, Say L, Seuc A, Broutet N (2013) Global estimates of syphilis in pregnancy and associated adverse outcomes: analysis of multinational antenatal surveillance data. PLoS Med 10: e1001396. 
25. Gomez GB, Kamb ML, Newman LM, Mark J, Broutet N, Hawkes SJ (2013) Untreated maternal syphilis and adverse outcomes of pregnancy: a systematic review and metaanalysis. Bull World Health Organ 91: 217-226.

26. World Health Organization (2011) Progress report 2011: Global HIV/AIDS response. World Health Organization. Available:

http://www.who.int/hiv/pub/progress_report2011/en/index.ht ml. Accessed: 10 October 2014.

27. Nair H, Arya G, Vidnapathirana J, Tripathi S, Talukder SH, Srivastava V (2012) Improving neonatal health in South-East Asia. Public Health 126: 223-226.

28. Mwapasa V, Rogerson SJ, Kwiek JJ, Wilson PE, Milner D, Molyneux ME, Kamwendo DD, Tadesse E, Chaluluka E, Meshnick SR (2006) Maternal syphilis infection is associated with increased risk of mother-to-child transmission of HIV in Malawi. AIDS 20: 1869-1877.

29. Hawkes SJ, Gomez GB, Broutet N (2013) Early antenatal care: does it make a difference to outcomes of pregnancy associated with syphilis? A systematic review and metaanalysis. PLoS One 8: e56713.

30. Zammarchi L, Borchi B, Chiappini E, Galli L, Brogi M, Sterrantino G, Trotta M (2012) Syphilis in pregnancy in Tuscany, description of a case series from a global health perspective. J Matern Fetal Neonatal Med 25: 2601-2605.

31. Mabey D, Peeling RW, Ustianowski A, Perkins MD (2004) Diagnostics for the developing world. Nat Rev Microbiol 2: 231-240.
32. Gu W, Yang Y, Wu L, Yang S, Ng LK (2013) Comparing the performance characteristics of CSF-TRUST and CSF-VDRL for syphilis: a cross-sectional study. BMJ Open 3: e002204.

33. Lafond RE, Lukehart SA (2006) Biological basis for syphilis. Clin Microbiol Rev 19: 29-49.

34. Terris-Prestholt F, Watson-Jones D, Mugeye K, Kumaranayake L, Ndeki L, Weiss H, Changalucha J, Todd J, Lisekie F, Gumodoka B, Mabey D, Hayes R (2003) Is antenatal syphilis screening still cost effective in sub-Saharan Africa. Sex Transm Infect 79: 375-381.

35. Fonck K, Claeys P, Bashir F, Bwayo J, Fransen L, Temmerman M (2001) Syphilis control during pregnancy: effectiveness and sustainability of a decentralized program. Am J Public Health 91: 705-707.

36. Schmid G (2004) Economic and programmatic aspects of congenital syphilis prevention. Bull World Health Organ 82: 402-409.

\section{Corresponding author}

Dr. A. Seval Ozgu Erdinc

Zekai Tahir Burak Women Health Care

Training and Research Hospital,

Talatpasa Bulvari, Hamamonu,

Ankara, Turkey

Phone: +903123065000

Fax: +90 3123124931

Email: sevalerdinc@gmail.com

Conflict of interests: No conflict of interests is declared. 